\title{
Die Crustaceen-Fauna des Alten Hafens zu Bremerhaven.
}

Von

W. Klie (Bremerhaven).

\section{Allgemeines.}

Während meines nunmehr vierjährigen Aufenthaltes in Bremerhaven konnte ich der Crustaceen-Fauna des Alten Hafens fortgesetzt meine Aufmerksamkeit widmen. Ich habe in allen Jahreszeiten Fänge mit dem Planktonnetz (Seidengaze Nr. 16) gemacht und bei diesen Gelegenheiten nie versäumt, auch die Enteromorpha-Rasen der Mauern und Balken abzustreifen, gelegentlich wurden auch Grundproben entnommen. Um ein möglichst umfangreiches und bezüglich der jahreszeitlichen Verteilung der Arten zuverlässiges Material bearbeiten zu können, beabsichtigte ich, im Jahre 1911 regelmäßig in Abständen von 14 Tagen Fänge zu machen und dabei gleichzeitig Tide, Salzgehalt und Temperatur zu notieren. Wenn auch in 18 Fällen diese Zeit ungefähr eingehalten werden konnte (die Zahl schwankt zwischen 11 bis 16 Tagen), so hat sich doch dieser Plan leider insofern nicht vollständig durchführen lassen, als in vier Fällen die Zwischenpausen wesentlich größer geworden sind: eine längere Abwesenheit im Sommer verursachte eine Unterbrechung von 7 Wochen, während im Winter eine starke Eisdecke die Ausdehnung der Pausen auf drei Wochen erforderte.

Der Alte Hafen ist in den Jahren 1827-30 angelegt und während der Jahre 1860-62 verbreitert worden, so daß jetzt bei einer Länge von $750 \mathrm{~m}$ die größte Breite $115 \mathrm{~m}$ beträgt. Da diese Breite jedoch nur im mittleren T'eile besteht, umfaßt er nur eine Wasserfläche von 7,2 ha. Die Tiefe beträgt bei mittlerem Hochwasser 7,06 m. Der im Laufe des Jahres 1912 verbreiterte Vorhafen hatte zur Zeit der Untersuchung eine Breite von $30-38 \mathrm{~m}$, seine Länge beträgt $270 \mathrm{~m}$, er Klie, Crastaceen-Fauna. Biol. Suppl. z. VI. Bd. 
schmiegt sich in schwacher Biegung der Mündung der Geeste an. ${ }^{1}$ ) Die Lage unmittelbar an der Geestemündung und die fast senkrecht die Einfahrt treffende Strömung der Weser bedingt in Verbindung mit der schmalen Schleusendurchfahrt $(11 \mathrm{~m})$ den verhältnismäßig geringen durchschnittlichen Salzgehalt des Wassers $(0,5 \%)$. Dieser ist übrigens einem fortwährenden Wechsel unterworfen, da die Spülkanäle der Schleuse bei jeder Tide ein Schwanken des Wasserstandes im Hafenbecken um $1 \mathrm{~m}$ und darüber bewirken, so daB also der Wechsel, der sich im Vorherrschen von Süßwasser bei Ebbe und im Hereindringen von Seewasser bei Flut im freien Strome stark bemerkbar macht, in ähnlicher, wenn auch etwas abgeschwächter Weise, auch im Hafen zu beobachten ist. Das spezifische Gewicht des Wassers wurde von mir durch Aräometerablesungen festgestellt. Herr Brockmann (Lehe) hatte die Güte, das von mir benutzte Aräometer mit einem Instrumente der Kgl. Biologischen Station auf Helgoland zu vergleichen, er prüfte ferner die Zuverlässigkeit der Spindel gegen Ende der Untersuchungszeit durch eine Kontrolltitration, sodab also die Angaben über den Salzgehalt als einwandfrei gelten dürfen. Aus dem durch die Aräometerbeobachtungen ermittelten spezifischen Gewicht wurde der Salzgehalt mit Hilfe der Hydrographischen Tabellen von M. Knudsen (Kopenhagen und Hamburg, 1911) abgeleitet. Ein Ansteigen des Salzgehaltes im Hochsommer und ein nachheriges Absinken im Herbst und Winter, wie es die Tabelle deutlich erkennen läBt, kann alljährlich beobachtet werden. Diese Erscheinung findet ihre Erklärung durch den verminderten Süßwasserzufluß und durch die gesteigerte Verdunstung während des Sommers. Ich glaube aber nicht, dab es zulässig ist, die in der Tabelle niedergelegten Erfahrungen des Jahres 1911, in welchem sich die ebengenannten Faktoren in außergewöhnlichem Grade wirksam erwiesen haben, zu verallgemeinern: in normalen Jahren dürfte eine über 7,5\% hinausgehende Konzentration des Hafenwassers selbst im Hochsommer zu den Seltenheiten gehören. Aus den Herbst- und Wintermonaten des Jahres 1910 stehen mir Angaben zum Vergleich zur Verfügung: betrug der Salzgehalt am 22. Oktober 1911 noch 8,13\% (siehe Tabelle), so war er an genau dem gleichem Tage des Jahres 1910 schon auf $3,35 \%$ gesunken, die entsprechenden Werte für den 17. Dez. 1911 und den 18. Dez. 1910 sind 6,32 und 1,80\%.

1) Diese Angaben sind entnommen aus: Rudloff, Claussen und Günther, Die Bremerhavener Hafen- und Dock-Anlagen und deren Erweiterung in den Jahren 1892-99. Sonderabdruck aus der Zeitschrift für Architektur und Ingenieurwesen. Hannover, 1903. 
Auf die quantitative Bestimmung der Crustaceen mußte von vornherein verzichtet werden, ich habe jedoch versucht, durch Anwendung der Zeichen $\mathrm{rr} \mathrm{r}+\mathrm{c}$ cc in der Tabelle die relative Häufigkeit schätzungsweise zum Ausdruck zu bringen. Allerdings war dieses Verfahren nur bei den beiden Arten durchführbar, die derart herrschend sind, daB die übrigen dagegen völlig zurücktreten, so daß derjenige, der nur gelegentlich Material entnimmt und den einzelnen Proben nicht eine ganz eingehende Untersuchung widmet, die Überzeugung gewinnen könnte, daß von Entomostraken nur Eurytemora affinis und Tachidius litoralis vorkommen. Tatsächlich ist auch nach fortgesetzten, eingehenden Beobachtungen der Eindruck bleibend, daß die Crustaceen-Fauna eine äußerst einförmige ist, denn nicht nur die wenigen SüBwasserformen, die als seltene Irrgäste aus dem Seengebiete der mittleren Geeste anzusehen sind, sondern auch die übrigen Arten, von denen man nach ihrem sonstigen Vorkommen annehmen könnte, daB sie im Brackwasser ihre Existenzmöglichkeit finden müBten, wurden stets nur in wenigen Exemplaren angetroffen. Die von Brockmann ${ }^{1}$ ) für die Diatomeen des Planktons der Wesermündung nachgewiesene Tatsache, daß das Brackwasser keine ihm ausschließlich angehörende Lebensformen ausbildet, wird durch die Ergebnisse der Untersuchung der CrustaceenFauna des Alten Hafens für die genannte Tiergruppe bestätigt. Uberwiegend sind es marine Arten, die eine weitgehende Verminderung des Salzgehaltes ertragen, sie treffen in der sogenannten Brackwasserzone mit den wenigen SüBwasserarten zusammen, die gegen eine gerınge Beimischung von Seewasser verhältnismäßig unempfindlich sind und bilden mit diesen eine Lebensgemeinschaft, deren Bestand an euryhalinen Arten zwar gesichert, deren Zusammensetzung aber infolge der durch die Gezeitenströmungen bewirkten Ergänzung von beiden Seiten her einem fortwährenden Wechsel unterworfen ist.

Neben der Untersuchung konservierter Fänge habe ich groBen Wert darauf gelegt, stets auch lebendes Material zur Verfügung zu haben. In flachen Schalen lassen sich an kühlen, dunklen Standorten die Cyclopiden und Harpacticiden wochenlang am Leben erhalten, so daß man in zweifelhaften Fällen die Entwicklung zur Geschlechtsreife abwarten und so bei der Identifikation mancher Arten völlig sicher gehen kann.

Herr Prof. Sars (Kristiania) hatte die Güte, die beiden in Betracht kommenden Schizopoden zu bestimmen; die Amphipoden sandte ich an

1) Brockmann, Das Plankton im Brackwasser der Wesermündung. Aus der Heimat - für die Heimat. Beiträge zur Naturkunde Nordwestdeutschlands, N. F., 1, Leipzig 1908. 
die Marine Biological Association in Plymouth, wo Frau E. W. Sexton die Bearbeitung des Materials vornahm, ihrer Vermittlung verdanke ich auch die Bestimmung der Isopoden durch Herrn Dr. Ch. Chilton (Port Chalmers, Neu-Seeland). Die Phyllopoden, Copepoden, Cirripedien und Decapoden habe ich selbst bestimmt, Ostracoden wurden auffallenderweise im Alten Hafen nicht gefunden.

In der nachfolgenden systematischen Aufzählung sollen alle die Angaben gemacht werden, die in der tabellarischen Ubersicht nicht Platz finden konnten. Zugleich sollen hier auch die Ergebnisse aller Fänge berücksichtigt werden, also auch derjenigen, die vor oder nach dem eigentlichen Beobachtungsjahr (März 1911 bis März 1912) gemacht worden sind, während die Tabelle nur über die Ergebnisse der Fänge des eben bezeichneten Zeitraumes Aufschluß gibt. Um Zweifel bezüglich der Identität der einzelnen Arten auszuschließen, findet sich hinter jedem Namen ein Hinweis auf ein wichtiges in Betracht kommendes Spezialwerk, dessen Titel unter der der fettgedruckten Ziffer korrespondierenden Nummer in dem der Arbeit beigefügten Literaturverzeichnis zu finden ist; die zweite Ziffer gibt die Seite des betreffenden Bandes an.

\section{Systematische Übersicht.}

\section{Phyllopoda.}

Sämtliche Cladoceren sind als gelegentliche Einwanderer zu betrachten, die meisten gehen sehr bald ein, nur die Bosmina-Arten und Chydorus sphaericus scheinen längere Zeit ausdauern zu können. Wenn nicht ausdrücklich das Gegenteil bemerkt worden ist, wurden die Tiere lebend gefangen.

$$
\text { Daphne pulex. De Geer. 10. } 79 .
$$

Am 10. November 1912 ein Männchen bei einem Salzgehalt von $0,94 \%$. Bosmina coregoni. Baird. 10. 284.

Die Notizen über das Vorkommen sind in der Tabelle enthalten.

$$
\text { Bosmina longirostris. 0. F. Müller. 10. } 225 .
$$

Am 30. November 1910 drei Exemplare $(S=1,29 \%$, am 9. Dezember 1910 ( $\mathrm{S}=1,38 \%$, am 13. Januar $1911(\mathrm{~S}=0,77 \%$ ) und am 13. Febr. $1913(\mathrm{~S}=0,26 \%)$ je ein Exemplar. Das Stück vom 13. Januar 1911 zeigte folgende Maße (auf Körperlänge 1000 umgerechnet): Körperhöhe 800. Autennenstiel 150. Endteil der Antenne 420. Schalenstachel 30, während die Messungen bei einem der Exemplare vom 30. November 1910 folgende Werte ergaben: Körperhöhe 840. Antennenstiel 126. Endteil der Antenne 272. Schalenstachel 0 . 
Camptocercus rectirostris. Schoedler. 10. 402.

Am 12. November $1910(\mathrm{~S}=3,08 \%)$ ein Weibchen tot.

Alonopsis elongata. Sars. 10. 434.

Am 1. November $1910(\mathrm{~s}=4,13 \%)$ ein Weibchen tot.

Alona rectangula. Sars. 10. 476. länge.

Am 28. Dezember $1910\left(\mathrm{~S}=1,16^{\circ} \%\right.$ ein Weibchen von $345 \mu$ KörperChydorus sphaericus. 0. F. Müller. 10. 561.

Außer den in der Tabelle angegebenen Funden kam diese Art noch vor am 3. Februar $1911(\mathrm{~S}=1,05 \%$, am 10. November $1912(\mathrm{~S}=0,94 \%)$ und am 13. Februar $1913(\mathrm{~S}=0,26 \%$.

\section{Copepoda.}

Diaptomus gracilis. Sars. 20. 92.

Diese in kleineren und größeren Gewässern Nordwestdeutschlands häufige Art gelangt gelegentlich ins Brackwasser, ohne sich jedoch dort längere Zeit halten zu können, wenigstens waren die zahlreichen Exemplare, die ich am 19. Juni 1911 im Hafen von Wremertief bei einem Salzgehalt von $4,1 \%$ erbeutete, schon nach wenigen Stunden sämtlich eingegangen. Im Alten Hafen wurde nur einmal, am 25. Oktober $1910(\mathrm{~S}=3,54 \%$ ein eiertragendes Weibchen gefangen.

\section{Eurytemora affinis. Poppe. 13. 56. 15. 184.}

Diese für die Gezeitenzone charakteristische Centropagiden-Art wurde in keinem Fange vermißt, die Maxima ihrer Entwicklung zeigen aber nicht die gleiche eindentige Beziehung zu einer bestimmten Temperatur, wie das bei Tachidius littoralis (siehe unten) der Fall ist, doch scheint das Optimum bei $14^{0} \mathrm{zu}$ liegen.

$$
\text { Cyclops viridis. Jurine, 11. } 8 .
$$

In zahlreichen Fängen gefunden, aber immer nur in wenigen Exemplaren.

$$
\text { Cyclops bicuspidatus. Claus. 11. } 11 .
$$

Bei C. bicuspidatus habe ich besonders auf das Vorkommen der Varietät mit 14. gl. Antennen (var. odessana Schmankewitsch $=$ lubbocki Brady) geachtet. Im Alten Hafen wurden nur Exemplare mit 17 gl. Antennen gefunden, dagegen kommt in einem kleinen brackischen Außendeichtümpel an der Franzosenschanze bei Blexen die Varietät mit 14 gl. Antennen massenhaft vor (festgestellt am 30. Dezember 1911, $\mathrm{S}=3,32 \%$ und am 3. April $1912, s=2,31 \%$. Demnach scheint die geringe Größe der Wohngewässer bei der in Rede stehenden Entwicklungshemmung von entscheidenderem Einflusse zu sein als der Salzgehalt. Vgl. zu dieser Frage: Blanchard und 
Richard (3. 515) - Can (4. 183) - Lilljeborg (11. 13) - Rehberg (18. 3) - Schmeil (22. 83) - Scourfield (23. 534).

Cyclops vernalis. Fischer. 11. 17.

Im März 1912 (siehe Tabelle) und am 13. Februar 1913 ( $=0,29 \%$ ) in wenigen Exemplaren gefangen.

Cyclops strenuus. Fischer. 11. 28.

Ebenfalls eine Frühjahrsform, die jedoch hänfiger beobachtet wurde als die vorige.

Cyclops Leuckarti. Claus. 11. 35.

Diese Art scheint sich in ganz schwach brackischem Wasser halten zu können, im Alten Hafen habe ich allerdings nur ein einziges Mal ein Weibchen gefangen, aber an zwei Stellen der unteren Geeste, Franzosenbrücke und Stauschleuse, wurde sie mehrfach in zahlreichen Exemplaren gefunden.

Cyclops oithonoides. Sars. 11. 42.

Im Alten Hafen wurde nur ein Weibchen von der typischen Form gefunden, während die Varietät hyalina Rehberg (17. 543-43) im Brackwasser der Geeste (Franzosenbrücke) beobachtet wurde.

$$
\text { Cyclops insignis. Claus. 11. } 52 .
$$

Das Vorkommen dieser Art, die Poppe für den Bremer Stadtgraben angibt (16. 520), im Alten Hafen ist nicht ganz sicher, da es mir nie gelang, geschlechtsreife Weibchen zu finden. Es traten jedoch mehrfach Männchen auf, die ich der Länge der Furkaläste und der Längenverhältnisse der Furkalborsten wegen nicht zu Cyclops strenuus stellen konnte, und die ich aus diesem Grunde auf $\mathrm{C}$. insignis beziehen möchte. (Furkaläste parallel, so lang wie die drei letzten Abdominalsegmente, längste Endborste fünfmal so lang als die innere.)

Cyclops languidoides. Lilljeborg. 11. 61.

C. languidoides scheint zu C. languidus Sars in demselben Verhältnisse zu stehen, wie C. odessanus bezw. lubbocki zu C. bicuspidatus (siehe oben). Die Form mit 16 gl. Antennen (languidus) habe ich in kleinen Moorgewässern mehrfach gefunden; aus dem Alten Hafen ist mir nur die Form mit $11 \mathrm{gl}$. Antennen (languidoides) bekannt geworden, allerdings habe ich diese auch gelegentlich im Süßwasser angetroffen.

Cyclops serrulatus. Fischer. 11. 81.

Ich habe nicht in allen Fällen die Zuweisung der aus dem Alten Hafen stammenden Exemplare zu Lilljeborgs Unterarten und Varietäten vornehmen können, bemerke aber, daß das einzige geschlechtsreife weibliche Stück aus dem Fange vom 17. März 1912 nach Lilljeborgs Terminologie als C. varius var, brachyurus zu bezeichnen wäre (11.90). 
Cyclops fimbriatus. Fischer. 11. 94.

Unmittelbar nach SchluB der Arbeitszeit eines Baggers gelang es mir, in dessen Nähe am 9. Dezember 1910 ein Weibchen dieser interessanten Bodenform zu erbeuten.

Cyclops aequoreus. Fischer. 11. 102.

Meine Mitteilung, die diese Art als neu für Deutschland anzeigte (8. 323), ist jetzt dahin zu berichtigen bezw. einzuschränken, daß C. aequoreus bisher in Norddeutschland nicht beobachtet worden war. Die später zu meiner Kenntnis gelangte Arbeit von Dahl (5. 178.) gibt die Art nämlich für die Neustädter Bucht und durch Sperr- und Fettdruck des Namens zugleich für die Unterelbe an. Das letztere ist sicher ein Irrtum, denn weder in den Fangprotokollen (155-160), noch in der systematischen Ubersicht (169-170) wird der C. aequoreus erwähnt, dagegen ist wohl nicht daran zu zweifeln, daß die Angabe für die Neustädter Bucht richtig ist. Die Art, von der allerdings immer nur wenige Exemplare gefangen wurden, scheint an unserer Küste weit verbreitet zu sein, denn ich fand sie, außer im Alten Hafen, sowohl im Brackwasser der Geeste, als auch an verschiedenen Stellen auf den Watten der Außenweser.

\section{Ectinosoma curticorne. Boeck. 21. 36 .}

Mehrfach beobachtet, aber immer nur in wenigen Exemplaren. Die Art scheint im Alten Hafen die Grenze ihrer Verbreitung gegen das SüBwasser zu erreichen, denn weiter stromaufwärts habe ich sie nie, stromabwärts dagegen häufig gefunden, wie denn auch Poppe (15. 198) und Timm (26. 381) ihr Vorkommen in der Jade bzw. bei Neuwerk angezeigt haben.

Attheyella pygmaea. Sars. 21. 202.

Süßwasserart, die nur zweimal in je einem Exemplare angetroffen wurde, in Gräben und Tümpeln in der Umgebung von Bremerhaven ist sie nicht selten.

Mesochra Lilljeborgi. Boeck. 21. 208.

Nächst Tachidis litoralis (siehe unten) kommt M. Lilljeborgi am regelmäßigsten im Alten Hafen vor, meistens jedoch nur vereinzelt, ein Auftreten in. größerer Anzahl habe ich in sechs Fällen, und zwar nur in den Wintermonaten, feststellen können.

Mesochra hirticornis. Scutt. 21. 210.

Von dieser außerordentlich interessanten Art wurden stets nur vereinzelte Exemplare gefangen. Sie ist in ihrem Vorkommen noch strenger auf die kältere Jahreszeit beschränkt als die vorige, denn in den Sommermonaten habe ich sie nie gefunden. Über das Vorkommen in Süßwasser und über die Berichtigung der Diagnose des Männchens vgl. 9. 25.

\section{Nitocra typica. Boeck. 21. 212.}

Von dieser Art haben mir überhaupt nur vier Exemplare, von denen eines, ein Männchen, aus dem Alten Hafen stammte, vorgelegen; sie scheint also an unserer Küste selten zu sein. 
W. Klie.

Nitocra spinipes, Boeck. 21. 213.

N. spinipes tritt, gegenüber der vorigen Art, mit ziemlicher Regelmäßigkeit auf, namentlich in den Wintermonaten; die Anzahl der Individuen bleibt aber stets gering, die Höchstzahl der Exemplare eines Fanges betrug fünf.

Laophonte nana. Sars. 21. 262.

Auch von dieser Art wurden nur vereinzelte Stücke gefangen und zwar in den Monaten November and Dezember; über ihre Verbreitung vgl. 9. 30.

Tachidius brevicornis. Lilljeborg. 21. 328 .

Von den beiden Tachidius Arten ist die vorliegende im Alten Hafen die seltenere und unbeständigere, die zudem stets nur in geringer Individuenzahl auftritt. Der Zusammendrängung der Fundnotizen in den Wintermonaten (siehe Tabelle) dürfte keine besondere Bedeutung beizumessen sein, denn ich habe an anderen Orten den T. brevicornis während des ganzen Jahres reichlich gefunden.

Tachidius litoralis. Poppe. 14. 149.

T. litoralis ist unter den Harpacticiden recht eigentlich die Charakterform des Alten Hafens. Bei geringer Entfaltung im Sommer, in einigen Fängen sogar fehlend, wird das Maximum durch eine Massenentwicklung in den Frühjahrs- und Herbstmonaten bei einer Wassertemperatur von $6-7^{0}$ erreicht.

\section{Cirripedia.}

Balanus improvisus. Darwin. 6. 250.

Diese Seepockenart findet sich im Alten Hafen gelegentlich an Balken und Holzwerk, wenn auch nicht so häufig wie an den Uferbauten des freien Stromes. Am 24. September 1911 (siehe Tabelle) wurde das Schwärmen der Nauplien beobachtet, die Stirnhörner waren bereits gestreckt, die meisten Exemplare entsprachen ungefähr dem von Münter und Buchholz (12. 32-38) beschriebenen und Tafel II, Fig. 27 abgebildeten Entwicklungsstadium.

\section{Amphipoda.}

Gammarus Zaddachi. Sexton. 24. 657. finden.

Sehr häufig und an geeigneten Stellen während des ganzen Jahres zu

Leptocheirus pilosus. Zaddach. 25. 630 .

Seltener als die vorige Art.

Microprotopus maculatus. Norman. 25. 604.

Im Alten Hafen wurde nur ein Exemplar gefunden, das zu bestimmen Herr Prof. G. 0. Sars die Güte hatte. Ein zweites Exemplar war in einem 
Fange von Eckwarderhörne (Jade) enthalten, wie Frau E. W. Sexton mir freundlichst mitteilte.

Corophium lacustre. Vanhöffen. 24. 664 .

Nächst Gammarus Zaddachi die häufigste der angeführten Arten.

\section{Isopoda.}

Sphaeroma rugicauda. Leach. 1. 408 .

Uber die beiden zur Bestimmung eingesandten Asseln schreibt Herr Dr. Ch. Chilton: "These seem to agree with the characters of Sp. rugicauda Leach better than with Sp. serratum Fabricius, but the specimens are hardly mature enough for certain determination."

\section{Schizopoda.}

Neomysis vulgaris. J. V. Thompson. 2. 339.

Diese Art, die noch bedeutend weiter stromaufwärts geht (sie wurde von mir auch bei Dedesdorf beobachtet), tritt im Sommer im Alten Hafen in Massen auf; nur während der Zeit der niedrigsten Wassertemperatur (Januar und Februar) wird sie völlig vermibt.

\section{Macropsis Slabberi. van Beneden. 19. 28.}

Diese zarte, glasartig durchsichtige Art konnte nur im Hochsommer und zu Beginn des Herbstes in fünf aufeinanderfolgenden Fängen nachgewiesen werden, es wurden in jedem Falle aber nur vereinzelte Exemplare gefunden.

\section{Decapoda.}

Crangon vulgaris. Fabricius. 2. 256. - 7 .

Gefangen wurden nur im Juni drei junge und im September ein völlig ausgewachsenes Exemplar; ebenfalls im September 1911 beobachtete ich an dem seichten Nordende des Hafens einen großen Schwarm von erwachsenen Tieren.

$$
\text { Carcinus maenas. Pennant. 2. } 76 .
$$

Von dieser am Weserdeiche und in den nördlicher gelegenen Häfen häufiger vorkommenden Krabbe fand ich im Alten Hafen nur ein kleines Exemplar. 
Tabellarische Zusammen-

\begin{tabular}{|c|c|c|c|c|c|c|c|c|c|c|c|c|c|c|c|c|}
\hline $\begin{array}{l}\underset{\Xi}{\Xi} \\
\underset{\Xi}{\Xi} \\
\Xi\end{array}$ & $\stackrel{+}{\Delta}$ & $\begin{array}{l}5 \\
0 \\
0 \\
0 \\
20 \\
5 \\
5 \\
5 \\
0 \\
0 \\
0 \\
0\end{array}$ & 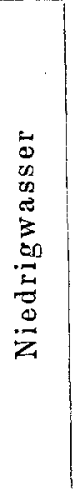 & 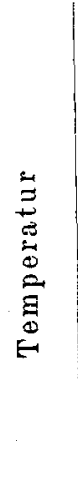 & 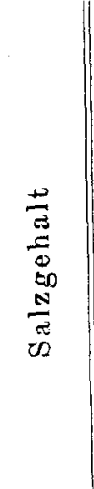 & 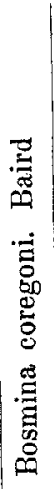 & 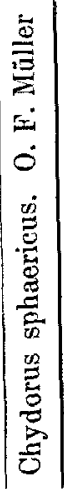 & 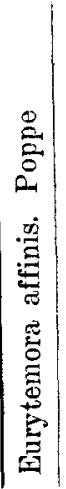 & 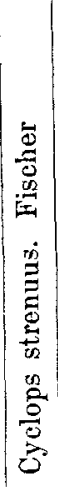 & $\begin{array}{c}0 \\
\frac{2}{3} \\
2 \\
\dot{0} \\
0 \\
0 \\
0 \\
0 \\
0 \\
0 \\
0 \\
0 \\
0 \\
0 \\
0 \\
0 \\
0 \\
0 \\
0\end{array}$ & 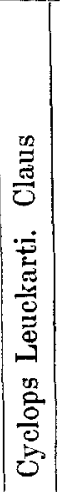 & 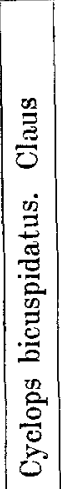 & 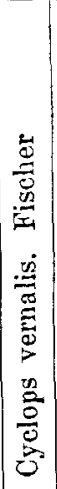 & 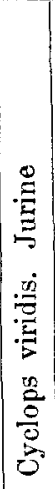 & 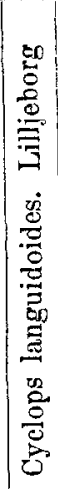 & 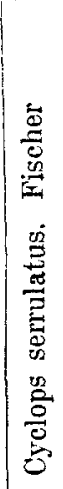 \\
\hline 1911. & & & & & & & & & & & & & & & & \\
\hline 16. III. & 745 & - & $9^{46}$ & 6,0 & 0,79 & - & - & + & • & & & & & - & . & \\
\hline 28. III. & 800 & - & $7^{14}$ & 6,2 & 0,41 & $\cdot$ & · & + & $\cdot$ & & & & & & - & \\
\hline 11. IV. & $10^{00}$ & $12^{31}$ & - & 6,8 & 1,41 & & - & $\mathrm{c}$ & • & & - & & & & $\cdot$ & \\
\hline 5. V. & $9^{15}$ & $6^{14}$ & - & 11,8 & 2,71 & & & $\mathrm{c}$ & • & $\cdot$ & & & & - & & \\
\hline 18. V. & $10^{35}$ & - & $11^{35}$ & 14,6 & 3,17 & & . & $\mathrm{ce}$ & • & & & - & & - & & \\
\hline 8. VI. & $10^{25}$ & $11^{24}$ & - & 17,6 & 6,38 & & & c & & & & & & & & \\
\hline 25. VI. & $11^{00}$ & $12^{38}$ & - & 17,6 & 5,11 & & & +1 & & & & & & & & \\
\hline 13. VIII. & $10^{25}$ & - & $10^{58}$ & 22,8 & 10,82 & & & c & & & & & & & & \\
\hline 27. VIII. & $10^{30}$ & - & $11^{02}$ & 19,4 & 12,06 & & & $\mathrm{c}$ & & & & & & & & \\
\hline 10. IX. & $10^{00}$ & - & 958 & 17,6 & 12,64 & & & c & & & & & & & & \\
\hline 24. IX. & $10^{00}$ & - & 956 & 15,8 & 11,92 & & & $\mathrm{c}$ & & & & & & & & \\
\hline 8. X. & $9^{00}$ & - & $8^{55}$ & 12,2 & 8,99 & & & + & & & & & & & & \\
\hline 22. X. & $8^{00}$ & - & $8^{48}$ & 10,8 & 8,13 & & & + & & & & & & & & \\
\hline 6. XI. & $10^{30}$ & - & $8^{17}$ & 7,8 & 10,31 & & & + & & & & & & & & \\
\hline 20. XI. & $8^{30}$ & - & $8^{18}$ & 7,0 & 8,47 & & & + & & & & & & & & \\
\hline 3. XII. & $10^{20}$ & $11^{24}$ & - & 3,8 & 6,05 & & & $r$ & & & & & & & & \\
\hline 17. XII. & $10^{30}$ & $11^{30}$ & - & 3,8 & 6,32 & & & + & & & & & & & & \\
\hline 31. XII. & $9^{1 \check{2}}$ & 921 & - & 4,8 & 2,43 & & & $\mathbf{r}$ & & & & • & & & & \\
\hline 1912. & & & & & & & & & & & & & & & & \\
\hline 21.1. & $8^{20}$ & - & $10^{00}$ & 0,9 & 1,37 & & & + & - & & & & & & & \\
\hline 6. II. & 845 & - & $11^{16}$ & 0,8 & 2,30 & & & $r$ & & & & & & & & \\
\hline 18. II. & 915 & - & 908 & 1,3 & 1,43 & & & $\mathrm{rr}$ & & & & & & & & \\
\hline 3. III. & $9^{15}$ & - & $8^{45}$ & 7,1 & 1,02 & & & $\mathrm{r}$ & - & & & • & - & $\cdot$ & & \\
\hline 17. III. & $8^{35}$ & - & $8^{02}$ & 7,2 & 0,76 & & & + & & & & . & & & & \\
\hline
\end{tabular}

\section{Literaturverzeichnis.}

1. Bate and Westwood, A History of the British sessile-eyed Crustacea 1868, Vol. II.

2. Bell, A History of the British stalk-eyed Crustacea. 1853. 
stellung der Fangergebnisse.

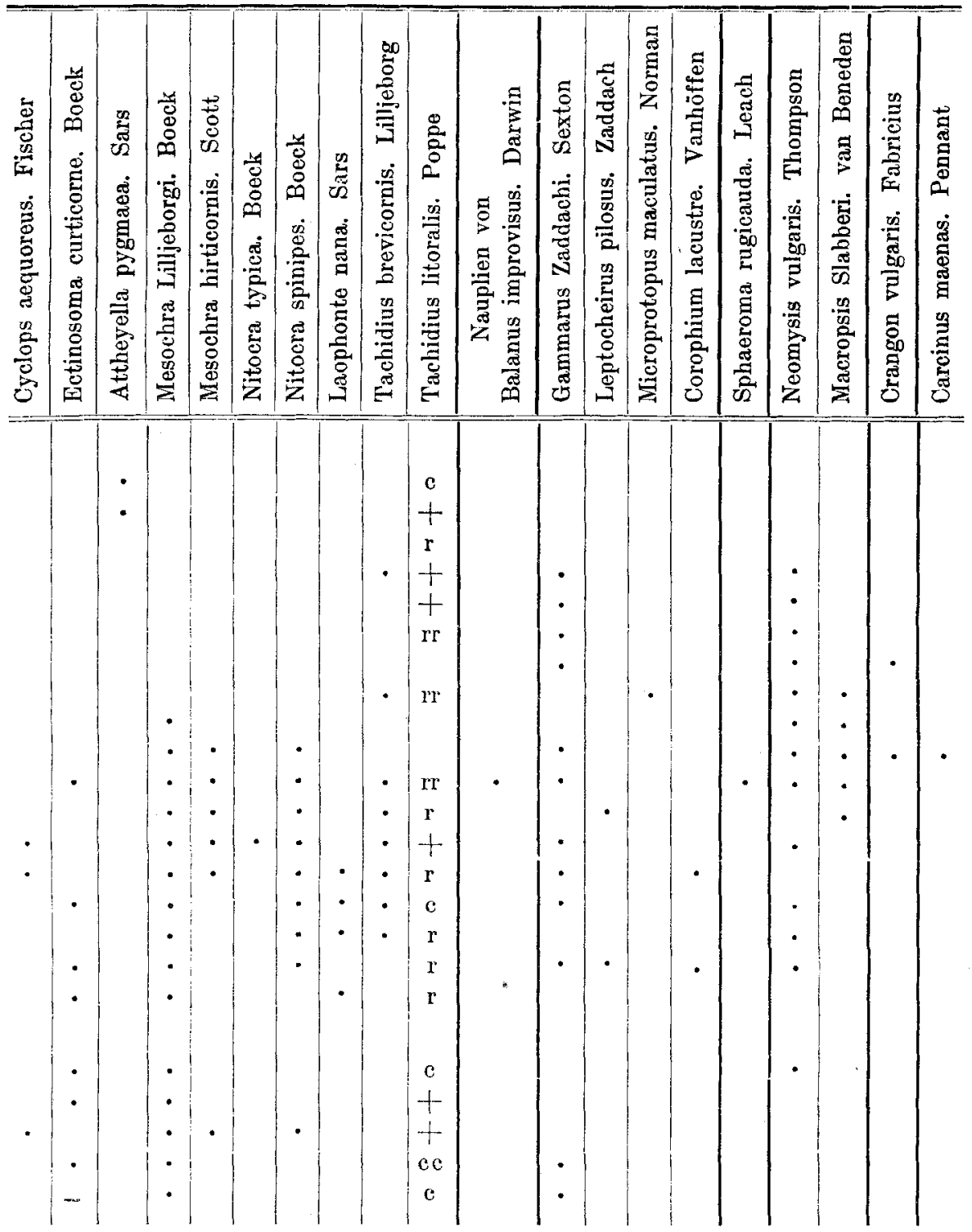

3. Blanchard et Richard, Faune des lacs salés d'Algérie. Mémoires de la société zoologique de France 1891, T. IV.

4. Canu, Les Copépodes du Boulonnais. 1892.

5. Dahl, Untersuchung über die Tierwelt der Unterelbe. 6. Bericht d. Komm. z. wissenschaftl. Unters. d. deutschen Meere, 17.-21. Jahrg., $1887-1891$. 
6. Darwin, A monograph of the Sub-Class Cirripedia. Vol. II, Balanidae, 1854.

7. Ehrenbaum, Zur Naturgeschichte von Crangon vulgaris. Fabr. Sonderbeilage zu d. Mitteil. d. Sektion f. Küsten- u. Hochseefischerei. 1890.

8. Klie, Zwei bemerkenswerte Entomostrakenfunde bei Bremerhaven. Archiv f. Hydrobiol. u. Planktonkunde 1911-12, Bd. VII.

9. Klie, Die Copepoda Harpacticoida des Gebietes der Unter- und $A u B e n w e s e r$ und der Jade. Separate Schriften des Vereins f. Naturkunde an der Unterweser 1913, III.

10. Lilljeborg, Cladocera Sueciae. Nova acta regiae societatis scientiarum Upsaliensis 1900, Vol. XIX.

11. Lilljeborg, Synopsis specierum huc usque in Suecia observatarum generis Cyclopis. Konglia Svenska Vetenskaps-Akademiens Handlingar 1901-02, Bd. XXXV.

12. Münter und Buchholz, Uber Balanus improvisus Darwin var. gryphicus Münter. Mitteil. aus d. naturw. Vereine v. Neu-Pommern $\mathfrak{u}$. Rügen $1869, \mathrm{Bd}$. I.

13. Poppe, Uber eine neue Art der Calaniden-Gattung Temora Baird. Abhandl. herausgeg. v. naturw. Vereine zu Bremen, Bd.VII, 1880.

14. Poppe, Uber einen neuen Harpacticiden. Daselbst.

15. Poppe, Die freilebenden Copepoden des Jadebusens. Daselbst, 1885, Bd. IX.

16. Poppe, Notizen zur Fauna der Süßwasserbecken des nordwestlichen Deutschland mit besonderer Berücksichtigung der Crustaceen. Daselbst 1889, Bd. X.

17. Rehberg, Beitrag zur Kenntnis der freilebenden SüBwasserCopepoden. Daselbst 1880, Bd. VI.

18. Rehberg, Beiträge zur Naturgeschichte niederer Crustaceen (Cyclopiden und Cypriden). Daselbst 1885, Bd. IX.

19. Sars, Arkiv f. Mathematik og Naturvidenskab. 1887, Bd. II.

Sars, An Account of the Crustacea of Norway.

20. Vol. IV. Calanoida. 1903.

21. Vol. V. Harpacticoida. 1911.

22. Sehmeil, Deutschlands freilebende SüBwasser Copepoden. Bibliotheca zoologica 1892, Heft 11, Cyclopidae.

23. Scourfield, Synopsis of the known species of British freshwater Entomostraca. Part. II. Copepoda. Journ. of the Quekett Micr. Club 1903, Ser. 2, Vol. VIII.

24. Sexton, Some Brackish-water Amphipoda from the mouths of the Weser and the Elbe, and from the Baltic. Proceedings of the Zoological Society of London. 1912.

25. Stebbing, Amphipoda I. Gammaridea. Das Tierreich. Lieferung 21. 1906.

26. Timm, Copepoden und Cladoceren. Beiträge zur Fauna der südöstlichen und östlichen Nordsee IV. Wissenschaftl. Meeresuntersuchungen 1894, N. F., Bd. 1. 